\title{
Directory of Certified Deciduous Fruit and Nut Crop Nurseries in Florida ${ }^{1}$
}

\author{
Peter C. Andersen, Robert C. Hochmuth, Sean R. McCoy, and Lei Lani L. Davis²
}

The sustainability of fruit and nut crops as a farm enterprise is dependent on the proper selection of a species and cultivar in relation to the specific region where the crops are to be grown (http://edis.ifas.ufl.edu/mg367). Florida represents several climatic regions. The proper selection of species and cultivars is probably the most important decision that determines the success or failure of a farm enterprise.

The University of Florida has released many fruit crops, including peaches/nectarines (http://www.hos.ufl.edu/ extension/stonefruit/stone-fruit-varieties), plums (http:// edis.ifas.ufl.edu/hs250), rabbiteye and southern highbush blueberries (http://edis.ifas.ufl.edu/ac031), muscadine grapes (http://edis.ifas.ufl.edu/hs100 and http://edis.ifas.ufl. edu/hs401), bunch grapes (http://edis.ifas.ufl.edu/mg105), an apple (http://edis.ifas.ufl.edu/hs764), and a pear. All of these releases have a low chilling requirement, which is critical to all growing regions of Florida. Many of these UF specialty crop cultivars ripen in early spring during a market window with little or no worldwide competition. Site selection is extremely important for successful production of most fruit and nut crops in Florida. Potential orchard sites should have soil that is well drained and slightly sloped for cold air drainage.

A number of cultivars have been developed for North, Central, and South Florida, and a continued emphasis for the breeding program is to increase the diversity of germplasm for the entire state. However, small and large producers seldom are aware of, nor can they find, these Florida-adapted cultivars of fruit and nut crops from local nurseries. Many of the UF cultivars are patented through the Florida Foundation Seed Producers, Inc. (FFSP, http:// ffsp.net), and bud wood is made available to those nurseries that purchase licenses. It is important to buy patented varieties from licensed nurseries. FFSP is a nonprofit corporation and direct support organization of the University of Florida. FFSP works closely with the Florida Agricultural Experiment Station (FAES), an agricultural and natural resources research program of the University of Florida's Institute of Food and Agricultural Sciences (UF/IFAS).

According to a 2007 agricultural census, $91 \%$ of all farms in the United States are classified as small farms (gross cash farm income $<\$ 250,000$ ). Likewise, more than $90 \%$ of all Florida farms are small farms. Florida is unique, however, in that it is one of the few states that showed an increase in the total number of farms in the 2007 census. This $8 \%$ increase in the total number of farms was essentially all in the small farm category. Small farmers in Florida have great opportunities because of the large urban centers, the mild climate, and abundant natural resources available. Most small farmers choose to directly market their products to the consumer to maximize profits. A diversity of fresh products to offer, such as fruits, nuts, and vegetables, generally is preferred at most direct-market venues. Some direct marketing strategies include farmer's markets, on-farm

1. This document is HS1218, one of a series of the Horticultural Sciences Department, Florida Cooperative Extension Service, Institute of Food and Agricultural Sciences, University of Florida. Original publication date April 2013. Visit the EDIS website at http://edis.ifas.ufledu.

2. Peter C. Andersen, professor, Horticultural Sciences Department; Robert C. Hochmuth and Sean R. McCoy, multi-county Extension agents, Live Oak; and Lei Lani L. Davis, agricultural technician, Live Oak; Florida Cooperative Extension Service, Institute of Food and Agricultural Sciences, University of Florida, Gainesville, FL 32611

The Institute of Food and Agricultural Sciences (IFAS) is an Equal Opportunity Institution authorized to provide research, educational information and other services only to individuals and institutions that function with non-discrimination with respect to race, creed, color, religion, age, disability, sex, sexual orientation, marital status, national origin, political opinions or affiliations. U.S. Department of Agriculture, Cooperative Extension Service, University of Florida, IFAS, Florida A\&M University Cooperative Extension Program, and Boards of County Commissioners Cooperating. Nick T. Place, Dean 
sales, roadside stands, subscription sales, communitysupported agriculture (CSA), U-pick, and sales directly to restaurants or institutions, such as schools (http://edis.ifas. ufl.edu/fy597).

Florida is poised to increase the number, sustainability, and profitability of small farms with increased access to new and improved cultivars, further exploitation of market windows, the development of new information and technologies on culture, and management and pest control, which includes insect monitoring, the use of trap cropping, safer targeted pesticides, and biological control. UF/IFAS is conducting research throughout the state to identify the key limiting factors to sustainability and profitability of low-chill fruit and nut crops by evaluating new cultivars, developing new production and management strategies, and using traditional and novel marketing dissemination methods.

Florida's subtropical climate is suitable for the culture of numerous fruit and nut trees. Certain species and cultivars can be grown successfully in Florida with a minimum of inputs (well adapted to Florida) or only with the investment of substantial inputs (marginally adapted), while others cannot be grown at all (non-adapted). Agricultural sustainability is largely a function of identifying and selecting adapted species/cultivars for a given geographic region. Proper crop selection results in minimal inputs, such as pesticides, irrigation, fertilizer, and labor. Sustainable crops are those with minimal inputs and consistent outputs, including yield, fruit quality, and minimal environmental impact. Crops that meet these criteria are candidates for organic practices, small farms with limited resources, and/ or a desire to avoid extensive inputs. However, there is not always a good correlation between profitability and sustainability. Other crops may be less sustainable (southern highbush blueberry) but still be attractive because of high potential profitability. Cultivar selection is the orchard's foundation. Appropriate cultivar selection minimizes inputs and maximizes profits.

This publication was developed to assist farmers in locating recommended cultivars of fruit and nut crops from Florida nurseries. The nurseries listed in Table 1 offer fruit and nut trees and are listed as certified nurseries with the Florida Department of Agriculture and Consumer Services (http:// www.freshfromflorida.com/pi/plantinsp/nursery_directory/ nur_directory.html). The nursery certification process is regulated by the Florida Department of Agriculture and Consumer Services (http://www.freshfromflorida.com/ pi/plantinsp/docs/brief_summary.pdf). Many nurseries listed in Table 1 only offer a few cultivars, while others offer several cultivars of many fruit or nut crops. Table 2 lists fruit and nut cultivars that University of Florida Extension specialists currently recommend or suggest on a trial basis. Maintaining an up-to-date list of cultivar inventory at each nursery is very challenging, so contact the nurseries directly to determine current inventory, or view their websites if available. 
Table 1. Directory of certified Florida nurseries offering fruit and nut crop trees

\begin{tabular}{|c|c|}
\hline Nursery business listing & Crops \\
\hline $\begin{array}{l}\text { Adam's Plants } \\
8135 \text { Southern Blvd. } \\
\text { West Palm Beach, FL } 33411 \\
\text { Phone: (561) 793-4303 } \\
\text { adamsplantsinc@yahoo.com } \\
\text { http://www.adamsplants.com }\end{array}$ & Banana, citrus, fig, muscadine grape, peach, plum \\
\hline $\begin{array}{l}\text { Agri-Starts } \\
1728 \text { Kelly Park Rd. } \\
\text { Apopka, FL } 32712 \\
\text { Phone: (407) 889-8055 } \\
\text { Fax: (407) 889-2523 } \\
\text { info@agristarts.com }\end{array}$ & $\begin{array}{l}\text { Banana, blackberry, southern highbush blueberry, rabbiteye } \\
\text { blueberry, fig, muscadine grape, peach, raspberry }\end{array}$ \\
\hline $\begin{array}{l}\text { Berry Blue Farm \& Nursery } \\
2903 \text { W 60th Hwy. } \\
\text { Plant City, FL } 33567 \\
\text { Phone: (813) 508-1104 } \\
\text { http://www.berrybluefarms.com/ }\end{array}$ & Southern highbush blueberry \\
\hline $\begin{array}{l}\text { Blue Gold Growers } \\
5645 \text { WO Griffin Rd. } \\
\text { Plant City, FL } 33567 \\
\text { Phone: (813) 748-5817 }\end{array}$ & Southern highbush blueberry \\
\hline $\begin{array}{l}\text { Bluestar Nursery } \\
8115 \text { SE US Hwy. } 301 \\
\text { Hawthorne, FL } 32615 \\
\text { Phone: (352) 481-3300 }\end{array}$ & Southern highbush blueberry, rabbiteye blueberry \\
\hline $\begin{array}{l}\text { Brite Leaf Citrus Nursery, LLC } \\
480 \text { CR } 416 \text { S } \\
\text { Lake Panasoffkee, FL } 33538 \\
\text { Phone: (352) 793-6861 } \\
\text { Fax: (352) 793-3674 } \\
\text { order@briteleaf.com } \\
\text { http://www.briteleaf.com }\end{array}$ & Cold-hardy citrus \\
\hline $\begin{array}{l}\text { Carlton Myers Nursery \& Orchard } \\
14451 \text { NE CR } 339 \\
\text { Trenton, FL } 32693 \\
\text { Phone: (352) } 463-6602\end{array}$ & Persimmon \\
\hline $\begin{array}{l}\text { Chestnut Hill Tree Farm, Inc. } \\
15105 \text { NW 94th Ave. } \\
\text { Alachua, FL 32615 } \\
\text { Phone: (386) 462-2820 / (800) 669-2067 } \\
\text { Fax: (386) 462-4330 } \\
\text { chestnuthilltreefarm@gmail.com } \\
\text { http://chestnuthilltreefarm.com }\end{array}$ & $\begin{array}{l}\text { Apple, banana, blackberry, southern highbush blueberry, rabbiteye } \\
\text { blueberry, cold-hardy citrus, chestnut, fig, grape, nectarine, olive, } \\
\text { peach, pear, pecan, persimmon, plum, pomegranate }\end{array}$ \\
\hline $\begin{array}{l}\text { Dewar Nurseries, Inc. } \\
625 \text { W Keene Rd. } \\
\text { Apopka, FL } 32703 \\
\text { Phone: (800) 329-1188 } \\
\text { http://www.dewarnurseries.com }\end{array}$ & $\begin{array}{l}\text { Banana, blackberry, fig, southern highbush blueberry, muscadine } \\
\text { grape, pomegranate, kiwi }\end{array}$ \\
\hline $\begin{array}{l}\text { Dole Berry Company East / Mixon Farms } \\
3510 \text { State Rd. } 546 \text { East } \\
\text { Haines City, FL } 33844 \\
\text { Phone: (863) 294-8856 / } 4301 \\
\text { Fax: 863-439-4134 } \\
\text { Rhonda Richardson } \\
\text { Rhonda.Richardson@dole.com } \\
\text { By appointment only }\end{array}$ & Southern highbush blueberry \\
\hline
\end{tabular}




\begin{tabular}{|c|c|}
\hline Nursery business listing & Crops \\
\hline $\begin{array}{l}\text { Excalibur Fruit Trees } \\
5200 \text { Fearnley Rd. } \\
\text { Lake Worth, FL } 33467 \\
\text { Phone: (561) 969-6988 } \\
\text { Fax: (561) 641-3028 } \\
\text { sales@excaliburfruittrees.com } \\
\text { http://www.excaliburfruittrees.com }\end{array}$ & $\begin{array}{l}\text { Apple, banana, blackberry, southern highbush blueberry, fig, grape, } \\
\text { nectarine, peach, pear, persimmon, plum, pomegranate }\end{array}$ \\
\hline $\begin{array}{l}\text { Florida Hill Nursery } \\
\text { PO Box 530318 } \\
\text { Debary, FL } 32753 \\
\text { Phone: (386) 873-8064 } \\
\text { floridahillnursery@yahoo.com } \\
\text { http://www.floridahillnursery.com }\end{array}$ & $\begin{array}{l}\text { Banana, blackberry, southern highbush blueberry, fig, muscadine } \\
\text { grape }\end{array}$ \\
\hline $\begin{array}{l}\text { Going Bananas } \\
24401 \text { SW 197th Ave. } \\
\text { Homestead, FL } 33031 \\
\text { Phone: (305) 247-0397 } \\
\text { Fax: (305) 247-7877 } \\
\text { goingbananas@bellsouth.net } \\
\text { http://www.going-bananas.com }\end{array}$ & Banana \\
\hline $\begin{array}{l}\text { Green Sea Farms } \\
6360 \text { Johnston Rd. } \\
\text { Zolfo Springs, FL } 33890 \\
\text { Phone: (863) 604-3778 } \\
\text { Cindy Weinstein } \\
\text { Greenseafarms@yahoo.com } \\
\text { http://greenseafarm.com }\end{array}$ & Pomegranate \\
\hline $\begin{array}{l}\text { Green Scene Nursery } \\
7544 \text { Number Two Rd. } \\
\text { Howey, FL 34737 } \\
\text { Phone: (352) 324-2560 } \\
\text { Kali Frey } \\
\text { gsn@atlantic.net } \\
\text { http://greenscenenursery.com }\end{array}$ & Pomegranate \\
\hline $\begin{array}{l}\text { Green's Nursery } \\
\text { 5844 Round Lake Rd. } \\
\text { Apopka, FL } 32798 \\
\text { Phone: (407) 889-3611 } \\
\text { Fax: (407) 889-0204 } \\
\text { http://www.greensnursery.com } \\
\text { Ray and Ann Green (owners) }\end{array}$ & $\begin{array}{l}\text { Apple, banana, blackberry, southern highbush blueberry, chestnut, } \\
\text { fig, muscadine grape, mayhaw, nectarine, peach, pear, pecan, } \\
\text { persimmon, plum, pomegranate }\end{array}$ \\
\hline $\begin{array}{l}\text { Hopkins Nursery } \\
25355 \text { Shultz Grade } \\
\text { Immokalee, FL } 34142 \\
\text { Phone: (239) } 658-0370 \\
\text { Fax: (239) 658-0390 } \\
\text { http://hopkinstropicalfruitnursery.com }\end{array}$ & $\begin{array}{l}\text { Banana, blackberry, southern highbush blueberry, fig, muscadine } \\
\text { grape, nectarine, olives, peach, persimmon, plum, pomegranate }\end{array}$ \\
\hline $\begin{array}{l}\text { Island Grove Ag. Prod. Nursery Div. } \\
2600 \text { SE 193rd Ave. } \\
\text { Hawthorne, FL } 32640 \\
\text { Phone: (352) 481-5558 / (352) 274-3835 } \\
\text { Fax: (352) 481-5093 } \\
\text { bsheffield@islandgroveagproducts.com } \\
\text { http://www.islandgroveagproducts.com } \\
\text { Bert Sheffield }\end{array}$ & $\begin{array}{l}\text { Blackberry, southern highbush blueberry, rabbiteye blueberry, fig, } \\
\text { muscadine grape, nectarine, peach, pomegranate }\end{array}$ \\
\hline
\end{tabular}




\begin{tabular}{|c|c|}
\hline Nursery business listing & Crops \\
\hline $\begin{array}{l}\text { Just Fruits and Exotics } \\
30 \text { St. Frances St. } \\
\text { Crawfordville, FL } 32327 \\
\text { Phone: }(850) \text { 926-5644 } \\
\text { Fax: }(850) \text { 926-9885 } \\
\text { justfruits@hotmail.com } \\
\text { http://www.justfruitsandexotics.com }\end{array}$ & $\begin{array}{l}\text { Apple, banana, blackberry, southern highbush blueberry, rabbiteye } \\
\text { blueberry, cold-hardy citrus, chestnut, fig, grape, mayhaw, nectarine, } \\
\text { olive, peach, pear, pecan, persimmon, plum, pomegranate, walnut }\end{array}$ \\
\hline $\begin{array}{l}\text { Lee's Nursery, Inc. } \\
\text { US Hwy. } 27 \text { E., PO Box } 26 \\
\text { Branford, FL 32008 } \\
\text { Phone: (386) 935-1410 } \\
\text { Fax: (386) 935-3020 } \\
\text { LeeNur1@windstream.net } \\
\text { James and Linda Lee }\end{array}$ & $\begin{array}{l}\text { Apple, blackberry, southern highbush blueberry, rabbiteye blueberry, } \\
\text { chestnut, fig, muscadine grape, mayhaw, nectarine, olive, peach, pear, } \\
\text { persimmon, plum, pomegranate }\end{array}$ \\
\hline $\begin{array}{l}\text { Lora's Lovely Plants } \\
11206 \text { S US Hwy. } 441 \\
\text { Lake City, FL } 32025 \\
\text { Phone: (386) 590-0390 } \\
\text { Fax: (386) } 755-9728\end{array}$ & $\begin{array}{l}\text { Apple, southern highbush blueberry, citrus, fig, nectarine, olive, } \\
\text { peach, persimmon, plum }\end{array}$ \\
\hline $\begin{array}{l}\text { Lychee Tree Nursery } \\
3151 \text { S Kanner Hwy. } \\
\text { Stuart, FL } 34994 \\
\text { Phone: (772) 283-4054 } \\
\text { http://lycheetreenursery.com }\end{array}$ & Fig \\
\hline $\begin{array}{l}\text { Miller Blueberry Plantation \& Nursery } \\
394 \text { CR } 315 \text { South } \\
\text { Interlachen, FL } 32148 \\
\text { Phone: (386) 684-6362 } \\
\text { Fax: (386) 684-1812 } \\
\text { info@millerblueberry.com } \\
\text { http://www.millerplantnursery.com } \\
\text { Jimmy Miller }\end{array}$ & Southern highbush blueberry, rabbiteye blueberry \\
\hline $\begin{array}{l}\text { Oasis Tropical Plants and Trees } \\
\text { Springhill, FL } 34606 \\
\text { Phone: (734) 308-4264 } \\
\text { oasiscustomersalwaysfirst@yahoo.com } \\
\text { http://oasistropicalplantsandtrees4u.com }\end{array}$ & Banana, citrus, fig \\
\hline $\begin{array}{l}\text { Pebbledale Farms } \\
12729 \text { Amber Ln. } \\
\text { Lithia, FL 33547 } \\
\text { Phone: (863) 450-8202 } \\
\text { Tina Connell } \\
\text { tina.pebbledale@hughes.net }\end{array}$ & Southern highbush blueberry \\
\hline $\begin{array}{l}\text { Phillip Rucks Citrus Nursery, Inc. } \\
1697 \text { Rucks Dairy Rd. } \\
\text { P.O. Box } 1318 \\
\text { Frostproof, FL } 33843 \\
\text { Phone: (863) 635-1948 } \\
\text { Fax: (863) 635-7624 } \\
\text { rucksnursery@aol.com }\end{array}$ & Cold-hardy citrus, low-chill peach \\
\hline $\begin{array}{l}\text { Pine Island Nursery } \\
16300 \text { SW 184th St. } \\
\text { Miami, FL 33187 } \\
\text { Phone: (305) 233-5501 } \\
\text { Fax: (305) 233-5610 } \\
\text { info@pineislandnursery.com } \\
\text { http://www.tropicalfruitnursery.com }\end{array}$ & $\begin{array}{l}\text { Apple, southern highbush blueberry, fig, muscadine grape, nectarine, } \\
\text { olive, peach, persimmon, plum, pomegranate }\end{array}$ \\
\hline
\end{tabular}




\begin{tabular}{|c|c|}
\hline Nursery business listing & Crops \\
\hline $\begin{array}{l}\text { S.E.M. Nursery } \\
12328 \text { NW SR } 45 \\
\text { High Springs, FL } 32643 \\
\text { Phone: (386) } 454-8084 \\
\text { Art Striver - appointment only }\end{array}$ & $\begin{array}{l}\text { Banana, rabbiteye and southern highbush blueberry, chestnut, } \\
\text { fig, muscadine grape, kiwi, olive, peach, pear, persimmon, plum, } \\
\text { pomegranate, black walnut }\end{array}$ \\
\hline $\begin{array}{l}\text { Simple Life Farms } \\
8135 \text { N Brook Way } \\
\text { Citrus Springs, FL } 34433 \\
\text { Phone: (352) 302-9559 }\end{array}$ & Southern highbush blueberry \\
\hline $\begin{array}{l}\text { Simpson Nurseries LAA } \\
\text { PO Box } 160 \\
\text { Monticello, FL } 32345 \\
\text { Phone: (800) 874-3571 / (229) 224-8242 } \\
\text { Fax: (850) 997-2518 } \\
\text { Chip Culbreth } \\
\text { http://simpsonnurseries.com }\end{array}$ & $\begin{array}{l}\text { Apple, blackberry, rabbiteye blueberry, fig, bunch and muscadine } \\
\text { grape, nectarine, peach, pear, pecan, persimmon, plum, pomegranate }\end{array}$ \\
\hline $\begin{array}{l}\text { Spyke's Grove } \\
7250 \text { Griffin Rd. } \\
\text { Davie, FL 33314 } \\
\text { Phone: (954) 583-0426 } \\
\text { oranges@att.net } \\
\text { http://spykestropicalnursery.com }\end{array}$ & $\begin{array}{l}\text { Apple, banana, blackberry, southern highbush blueberry, rabbiteye } \\
\text { blueberry, cold-hardy citrus, chestnut, fig, muscadine grape, } \\
\text { nectarine, olive, peach, persimmon, plum, pomegranate }\end{array}$ \\
\hline $\begin{array}{l}\text { True Blue Plants } \\
8701 \text { Rhodes Rd. } \\
\text { Hudson, FL } 34669 \\
\text { Phone: (877) 237-5268 } \\
\text { Fax: (727) 674-4067 } \\
\text { http://www.trueblueplants.com }\end{array}$ & Southern highbush blueberry, rabbiteye blueberry \\
\hline $\begin{array}{l}\text { Twigs and Berries Nursery } \\
300 \text { Cheney Hwy. } \\
\text { Titusville, FL 32783-5712 } \\
\text { Phone: (407) 832-8326 } \\
\text { Steve Kopacz } \\
\text { steve@twigsandberries.net } \\
\text { miraclefruitman@aol.com } \\
\text { http://www.twigsandberries.net }\end{array}$ & $\begin{array}{l}\text { Apple, banana, blackberry, southern highbush blueberry, cold-hardy } \\
\text { citrus, chestnut, fig, muscadine and bunch grape, nectarine, olive, } \\
\text { peach, pear, persimmon, plum, pomegranate }\end{array}$ \\
\hline
\end{tabular}


Table 2. Recommended fruit and nut species and cultivars grown in North and North Central Florida

\begin{tabular}{|c|c|c|}
\hline Species & Recommended cultivars & Recommended for trial \\
\hline Pecan (Carya illinoensis) & $\begin{array}{l}\text { Cape Fear, Elliott, Curtis, Stuart, Sumner, } \\
\text { Moreland }\end{array}$ & $\begin{array}{l}\text { Amling, Caddo, Excel, Forkert, Kanza, } \\
\text { Desirable, Pawnee, Lakota, Gafford, } \\
\text { Headquarters, Baby B, Apalachee, Creek, } \\
\text { Giftpack, Oconee, Byrd, Mandan, Kiowa }\end{array}$ \\
\hline Peach (Prunus persica) & $\begin{array}{l}\text { North Florida: Flordacrest, Flordaking, } \\
\text { Gulfking, Gulfcrest, Gulfcrimson } \\
\text { North Central Florida: Flordabest, Flordacrest, } \\
\text { Gulfking, UFBeauty } \\
\text { Central Florida: UFSun, UFOne, Flordaprince, } \\
\text { TropicBeauty }\end{array}$ & $\begin{array}{l}\text { North Florida: Gulfprince, GulfAtlas, Gulfsnow, } \\
\text { UFGlo } \\
\text { North Central Florida: UF2000, UF Blaze, UFO, } \\
\text { UFSharp, TropicSnow } \\
\text { Central Florida: UFBest, UFBeauty, Flordaglo, } \\
\text { UFGold }\end{array}$ \\
\hline Nectarine (Prunus persica) & $\begin{array}{l}\text { North Florida: Suncoast } \\
\text { North Central Florida: Sunbest, Sunmist }\end{array}$ & $\begin{array}{l}\text { North Florida: Sunmist } \\
\text { North Central Florida: UFQueen, UFRoyal }\end{array}$ \\
\hline Plum (Prunus salicina) & Gulfbeauty, Gulfblaze, Gulfrose & $\begin{array}{l}\text { For extreme North Florida only: Au-Homeside, } \\
\text { Au-Producer, Au-Roadside, Au-Rosa, Au- } \\
\text { Rubrum, Byrongold, Excelsior, Ozark Premier, } \\
\text { Robusto, Segundo }\end{array}$ \\
\hline $\begin{array}{l}\text { Southern highbush blueberry (Vaccinuum } \\
\text { corymbosum } \times \text { V. darrowi) }\end{array}$ & Emerald, Farthing, Jewel & $\begin{array}{l}\text { For Central Florida only: Snowchaser, } \\
\text { Springhigh, Primadonna, Meadowlark, } \\
\text { Scintilla } \\
\text { For North Florida only: Georgiagem, Oneal, } \\
\text { Rebel, Sweetcrisp } \\
\text { For North Central Florida only: Springhigh, } \\
\text { Meadowlark, Primadonna, Snowchaser }\end{array}$ \\
\hline Rabbiteye blueberry (Vaccinium virgatum) & $\begin{array}{l}\text { Austin, Climax, Brightwell, Powderblue, } \\
\text { Premier, Savory }\end{array}$ & $\begin{array}{l}\text { For North Florida only: Arapaho, Ira, } \\
\text { Ochlocknee, Yadkin }\end{array}$ \\
\hline Blackberry (Rubus spp.) & $\begin{array}{l}\text { North Florida: Ouachita, Natchez (both } \\
\text { thornless), Kiowa (thorny) }\end{array}$ & $\begin{array}{l}\text { North Florida: Apache, Arapaho, Navaho (all } \\
\text { thornless), Tupy (thorny). } \\
\text { North Central and Central Florida: Brazo, } \\
\text { Oklawaha, Flordagrand, Tupy }\end{array}$ \\
\hline Muscadine grape (Vitis rotundifolia) & $\begin{array}{l}\text { Fresh-market grapes: Black Beauty, Black Fry, } \\
\text { Fry, Granny Val, Ison, Pam, Pineapple, Tara, } \\
\text { Polyanna, Southern Home, Summit, Supreme, } \\
\text { Sweet Jenny } \\
\text { Processed grapes: Alachua, Carlos, Noble, } \\
\text { Welder }\end{array}$ & $\begin{array}{l}\text { Fresh-market grapes: Majestic, Southern } \\
\text { Jewel, Delicious, Eudora, African Queen, Big } \\
\text { Red, Darlene, Doreen, Early Fry, Florida Fry, } \\
\text { Nesbitt, Lane } \\
\text { Processed grapes: Creek, Golden Isles }\end{array}$ \\
\hline Southern bunch grape (Vitis spp.) & Processed grape (wine): Blanc duBois & $\begin{array}{l}\text { Fresh-market grapes: Daytona, Orlando } \\
\text { Seedless, Emerald, Blue Lake } \\
\text { Processed grapes (wine): Suwannee, Stover, } \\
\text { Black Spanish, Conquistador }\end{array}$ \\
\hline Apple (Malus domestica)* & Anna, Dorsett Golden & Tropic Sweet \\
\hline Pear (Pyrus serotina)* & $\begin{array}{l}\text { Baldwin, Flordahome, Hood, Tenn, Kieffer } \\
\text { Orient, Carnes, Pineapple, Moonglo }\end{array}$ & Housi, Shinseeki, $20^{\text {th }}$ Century \\
\hline Oriental persimmon (Diospyros kaki) & Fuyu & $\begin{array}{l}\text { Izu, Matsumoto Wase Fuyu, Ichikikei Jiro, } \\
\text { Hana Fuyu, Jiro, Suruga }\end{array}$ \\
\hline Chestnut (Castanea mollisima) & $\begin{array}{l}\text { Seedlings which are best propagated from } \\
\text { large seeds }\end{array}$ & Carolina, Revival, Heritage, Williamette \\
\hline Mayhaw (Crataegus aestivalis)* & Insufficient data & $\begin{array}{l}\text { Lori, Lindsey, Big Red, Red and Yellow, } \\
\text { Heavy, Mason's Super Berry, T.O. Super Berry, } \\
\text { Highway Super Berry, Super Spur }\end{array}$ \\
\hline Fig $\left(\right.$ Ficus carica) ${ }^{*}$ & Brown Turkey, Black Spanish, Celeste & $\begin{array}{l}\text { Alma, Conadria, Jelly, Osborne Prolific, } \\
\text { Pasquale, Tena, Ventura, Champagne, } \\
\text { Green Ischia, Kadota, LSU Gold, LSU Purple, } \\
\text { Magnolia, O'Rourke, Tiger }\end{array}$ \\
\hline
\end{tabular}




\begin{tabular}{|c|c|c|}
\hline Species & Recommended cultivars & Recommended for trial \\
\hline Olives (Olea europaea)* & $\begin{array}{l}\text { Although Arbequina, Mission, and Manzanillo } \\
\text { appear adapted to North Florida, we have } \\
\text { insufficient data at this time. }\end{array}$ & $\begin{array}{l}\text { Arbequina, Mission, Manzanilla, Ascolana, } \\
\text { Lecino, Frontio, Coratina, Kalamara, Arbosana } \\
\text { Koroneiki }\end{array}$ \\
\hline Pomegranate (Punica granatum) & None (insufficient data at this time) & $\begin{array}{l}\text { Thomson, Cranberry, Don Summer South, } \\
\text { Don Summer North, Nikitski Ranni, Pink, King, } \\
\text { Eve }\end{array}$ \\
\hline Kiwi (Actindia deliciosa) & $\begin{array}{l}\text { None (A prior trial at North Florida Research } \\
\text { and Education Center based on the Hayward } \\
\text { cultivar was not successful.) }\end{array}$ & $\begin{array}{l}\text { Hayward, ZESPRI Green, ZESPRI Gold, ZESPRI } \\
\text { Organic }\end{array}$ \\
\hline Banana (Musa spp.) & $\begin{array}{l}\text { None (Bananas require cold protection and } \\
\text { usually require } 12-18 \text { months to produce a } \\
\text { crop.) }\end{array}$ & Cavendish (protection required) \\
\hline Satsuma (Citrus unshiu) & Owari, Brown Select, Kimbrough & Early St. Anne, Xie Shan \\
\hline Kumquat (Fortunella $\times$ crassifolia) & Meiwa, Nagami & Murumi, Hongkong \\
\hline
\end{tabular}

